\title{
DISCLAIMER
}

This reporl was prepased ax an account of work sponsored by an agency of the linited Siutes Government. Neither the United States Governmen nor any agency thereof. now any of their

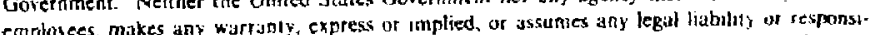
byils for the accuracy, completeness, or usefulness of any inforniation. apparalus, produce, or process disclosed, or represents that its use would not infritige privately uwned righls Reference herein to any specific commercisl product, process, of service by todde name, irademark. manufacturer, of otherwise does ant necessarily constitute or imply its endursement, recommendation. of Iavoring by the Unired States Gowernment or any agency thereof. The yews and opinions of guthors expressed herein do not necessatily state ar reflect thene of the United States Government or any agency thereof.

\section{Analysis of an Scrape-off Layer as a Electrostatic Barrier and Controlling the Particle aque for Reducing on the Large Major Radiu and Energy Losses}

\section{B. LaBombard, R. W, Conn}

\section{UCLA-PPG-1186}

December, 1988

\author{
Institute of Plasma and Fusion Research \\ Deparment of Mechanical, Aerospace and Research \\ University of California, Los Auclear Engineering \\ Los Angeles, CA, 90024 , US
}

A shortened version of this repon has been submitted for
publication to Plasma Physics and Controlled Fusion 
DISCAMER

This teport wat prepered at in sccount of work sponsored by an agency of the United Stutes Government. Neither the United States Government nor any agency thereof, nor any of uheir employees, makes any warranty, express or implied, or astumes eny legal liability of responsibility for the eccuracy. completeness, or usefulness of any information, apparatus, product, or process disclosed, or represents thet jts use would not infringe privelely owned rights. Refesence hertis to any specific commercial produch process, or service by trade name, trademert, manufucturer, or oherwise, daes not necessarily constitute or imply its endorsement, recommendation. or favoring by the United States Government or any agency theteof. The views and opinions of whorg exprested herein do not necessarily state or reflect those of the United States Govemment of any egency thereof.

$+$

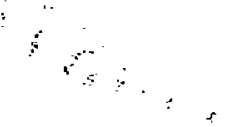




\section{Table of Contents}

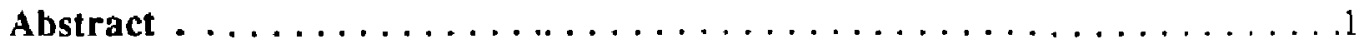

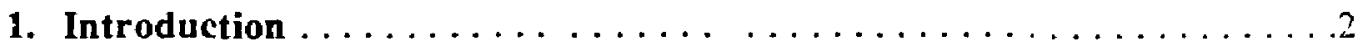

2. A Non-Ambipolar Transport Model for Biased Scrape-off Plasmas in Tokamaks . . . . . . . . . . . . . . . . . . . .

$2.1 \quad$ EXB and Electrostatic Barrier Approaches $\ldots \ldots \ldots \ldots \ldots \ldots$

2.2 Model for Cross-Field Plasma Transport in a Biased

Scrape-off Layer . . . . . . . . . . . . . . . . . . . s

A. Radial Fluxes and Currents ...................

B. Parallel Fluxes and Currents. ...................

C. BSOL Density and Potential Profiles. ...............

2.3 Comparison of Model with Results from the ALT-I

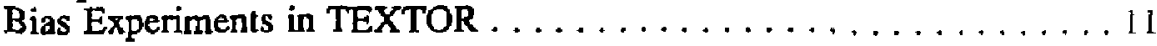

3. An $\mathbf{m}=1$ Electrostatic Barrier Scrape-off Layer (EBSOL) $\ldots \ldots \ldots \ldots \ldots 12$

$3.1 \mathrm{~m}=1$ versus $\mathrm{m}=0$ Biased Limiter Structures . . . . . . . . . . . . 12

3.2 Observations of Perpendicular Transport Asymmetries . . . . . . . . . 13

$3.3 \mathrm{~m}=1$ EBSOL Voltages and Currents. . . . . . . . . . . . . 13

A. Bias Voliages for $m=1$ EBSOL: Matching of Inside and

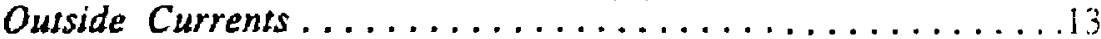

B. Bias Currents for $m=1$ EBSOL: Plasma Fluxes into the

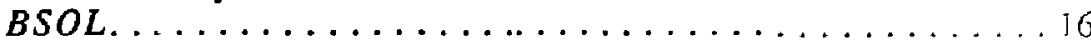

3.4 The $m=1$ EBSOL as an ExB Divertor . . . . . . . . . . . . . . . . 17

3.5 Refinements to the EBSOL Scheme: High Recycling Divertor, Other Biases . . . . . . . . . . . . . . . . . . . 0

3.6 Effect of 'Intrinsic Biases' on Transport Analysis and Transport Asymmetries. . . . . . . . . . . . . . 11

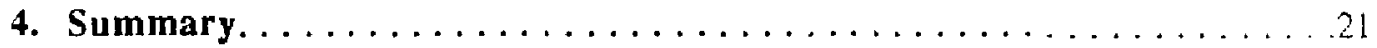

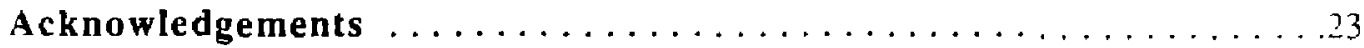

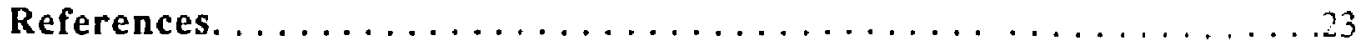

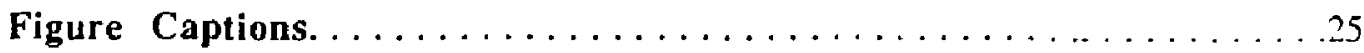

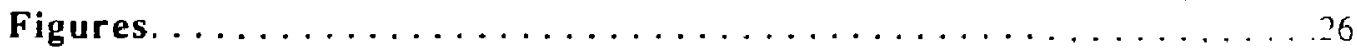




\title{
Analysis of an $m=1$ Electrostatic Barrier Scrape-off Layer as a Technique for Reducing and Controlling the Particle and Energy Losses on the Large Major Radius Edge of a Tokamak
}

\author{
B. LaBombard, R.W. Conn \\ Institute of Plasma and Fusion Research \\ Department of Mecharical, Aerospace and Nuclear Engineering \\ University of Califormia, Los Angeles \\ Los Angeles, CA 90024, USA
}

It is observed in many tokamaks that particle and heat fluxes from the core region are poloidally asymmetric, favoring higher cross field transport on the large major radius edge of the torus. We propose a novel technique that may allow one to control this asymmetric flux into the tokamak boundary plasma. The scheme principally involves the formation of a mobility limited transport layer or 'electrostatic barrier' to inhibit the flow of plasma into the boundary layer at large major radii, forcing plasma to exit on the small major radius side of the torus instead. At the same time, the technique produces an 'ExB divertor' effect, efficiently exhausting plasma and impurities within the boundary layer.

The implications of such a scheme are potentially important. By forcing plasma to exit on the inside half of the torus where the intrinsic cross-field transport is lower, the overall confinement characteristics of the central plasma may be improved. Furthermore, scrape-off plasma fluxes, subsequent recycling conditions, and their asymmetries at limiter, divertor, and wall structures can be actively controlled. An important feature of the technique is that it may be employed in both limiter and divertor configurations.

The first-order response of the scrape-off layer plasma to the applied bias is estimated in this report using a simple two fluid transport model which includes cross-field diffusion and mobility. It is shown that the influence of an applied bias on the scrape-of layer density e-folding length depends only on the relative magnitudes of cross-field ion and electron mobilities. Thus, even in the case of anomalous diffusion (Bohm) and classical mobility, a strong 'electrostatic barrier' may be maintained with applied potentials on the order of the electron temperature. The strength of the predicted resultant perpendicular electric field is large since it depends on the ratio of cross-field diffusion to mobility. Such a strong perpendicular electric field may be beneficial for two reasons: (1) ExB flows may be used to supplement or repiace plasma exhaust by a magnetic divertor system; and (2) strong perpendicular electric fields may reduce turbulent transport on the outside half of the boundary plasma thereby improving central piasma confinement. 


\section{INTRODUCTION}

The technique of externally imposing an electrical potential to limiter and wall surfaces to affect a change in edge plasma flows has been attempted in many tokamak devices [1-7]. Typically a potential is applied between the vacuum vessel wall and a limiter. These 'biased limiter' experiments have shown that the boundary layer or 'scrape-off plasma' fluxes can be significantly altered. In some cases the global particie confinement time can be changed. The mechanism responsible for local reductions or enhancements in the cross-field particle flux within the scrape-off layer can be viewed qualitatively as a formation of a 'potential barrier' to cross-field ion flow. It appears that a potential barrier of a height on the order of the electron temperature (or possibly ion temperature) is sufficient to markedly reduce the local cross-field transport. Consequently, imposing a bias of sufficient magnitude to a limiter or divertor surface relative to another object or the vacuum vessel wall surface can move the location of the active plasma-wall interaction region and thus change the global recycling coefficient.

As an extension of this technique, one might envision forming a controlled potential barrier all around the plasma periphery thereby affecting the loss rate of all particles originating from the core plasma. However, no physical arrangement of electrodes can be used to impose such a barrier on closed flux surfaces. One must resoit to non-electrode means such as the use of RF or high energy charged particle injection to affect a plasma-wall potential [3], or neutral beams to generate a VxB potential well. These techniques are relatively complicated, inefficient, and suffer from some undesirable side-effects such as impurity accumulation in the central plasma.

On the other hand, data from many tokamaks devices indicate that particle and energy fluxes from the plasma core are poloidally asymmetric, showing higher cross field transport on the large major radius edge of the torus [8,9]. Thus, it may not be necessary to form a potential barrier around the full periphery of the plasma but only on the outside half of the torus where the cross-field transport is high. This suggests the basic idea of this paper, namely, that two limiter or divertor 'electrodes' be used to impose an effective electrostatic barrier to cross-field ion flow. The arrangement would be similar in operation to a very large 'double probe', effectively biasing the scrape-off plasma (SOL) on the outside half of the torus positive relative to the SOL on the inside half of the torus. In this way, the last closed flux surface wculd acquire a plasma potential with a value intermediate between the potential of the two SOL plasmas, forming a potential barrier on the outside half of the torus. The imposed plasma potential perturbation need not propagate into the central plasma since it is only required that fluxes into the plasma boundary be controlled. In this respect, impurity accumulation problems associated with other bias schemes might be avoided. However, should the potential perturbation extend slightly onto closed flux surfaces, one may envision inducing an 'artificial $\mathrm{H}$-mode' by externally supplying the strong radial electric field that is believed to play an important role in $\mathrm{H}$-mode 
discharges [10]. In any case, the technique allows one to dynamically control the poloidal distribution of particle and heat fluxes within the scrape-off layer, regulating plasma exhaust and/or reducing asymmetries on limiter, divertor, and wall components.

In order to motivate the development and further discussion of such an electrostatic barrier scrape-off layer (EBSOL) concept, section 2 reviews some observations that have been previously reported in tokamak 'biased limiter' experiments and develops a simple model for cross-field plasma transport in a biased scrape-off layer. A qualitative comparison of the model is made with results from biased limiter experiments on TEXTOR. Section 3 introduces the EBSOL concept in detail, estimating particle fluxes and currents based on this transport model. Methods for implementation as a 'biased limiter' or a 'biased divertor' are considered. The impact of ExB flows on particle exhaust in the scrape-off layer is investigated. Possible refinements to the EBSOL technique are discussed including operation in a high recycling divertor and/or in conjunction with other bias schemes which one might envision in a tokamak. Finally, the idea that some asymmetries reported in tokamak scrape-off layers may be a consequence of 'intrinsic biasing' is considered. Section 4 summarizes the contents of this paper.

\section{A SIMPLE NON-AMBIPOLAR TRANSPORT MODEL FOR BIASED SCRAPE-OFF LAYER PLASMAS IN TOKAMAKS}

\subsection{EXB and Electrosiatic Barrier Approaches}

The effect of an applied electrical bias to limiter an wall components has been studied in a variety of toroidal discharges [1-7]. The general goal in these experiments is to change in some way the local transport properties of the edge plasma. Enhanced transport may lead to improved particle removal while reduced transport may improved particle confinement. Either of these conditions may be desirable for tokamak edge plasma control.

One can characterize the mechanisms responsible for the change in cross-field transport due to an applied D.C. electric field through a simplified transport model. For the case of ion transport, the cross-field plasma ion flux ( $\Gamma$ ) may be approximated as

$$
\Gamma=-D \nabla n+n \frac{E \times B}{B^{2}}+\mu n E+n \frac{B \times \nabla P_{i}}{q B^{2}}
$$

where $D$ and $\mu$ are effective cross field diffusion and mobility coefficients respectively and $\mathbf{E}$ is the electric field. The appropriate values for $D$ and $\mu$ are 
ultimately determined by the level of plasma turbulence and can be generally complicated functions of the local plasma conditions, including the local electric field itself. Nevertheless, eq. (1) serves to illustrate our discussion.

By the proper choice of electrode geometry, one may attempt to induce ExB fluxes and/or mobility-driven fluxes so as to affect the overall local paricle flux through eq. (1). The prospect of using ExB fluxes to modify the boundary layer was investigated most extensively by Strait et al, in connection with an 'ExB scraper' concept $[1,2]$. However, more recent limiter bias experiments [3-7] appear to rely on mobility-driven fluxes. In principle, the diffusion term can be completely cancelled by the mobility term by applying a sufficiently high electric field collinear with $\nabla n$. Assuming for the moment that the cross-field diffusion and mobility terms scale according to the Einstein relation, $D=\kappa T \mu / q$, the magnitude of the eiectric ricld required to stop the ion flux would be

$$
E \geq \kappa T / q \lambda_{n}
$$

where $\lambda_{n}$ is the density e-folding length. Thus a potential variation on the order of the plasma temperature over a distance of a density e-folding length would be required. If at some location eq. (2) is satisfied, one may consider that an elecurostatic barrier has been successfully formed, forcing a significant reduction in the ion flux. Of course this model predicts that in 'stoppering up' the local ion flux one must be willing to increase the local electron flux, given that the diffusion and mobility coefficients remain unchanged.

At first glance, it appears that this bias technique only serves to trade-off the local ion and electron transport properties and that, to a first approximation, the total convected energy remains unchanged. However, there is evidence that the turbulence that gives rise to the phemonenologically observed values for $D$ and $\mu$ also depends on $\mathbf{E}$. Consequently, an externally imposed $\mathbf{E}$ may affect the total convected energy through this link. Such a link is suggested in the simultaneous observations of improved central plasma confinement and formation of steep temperature and potential gradients in the edge plasma of $\mathrm{H}$-mode discharges. Direct measurements on TEXT (5) show that particle transport due to electrostatic turbulence is reduced during a limiter biasing arrangement that imposes a radial electric field parallel to $\nabla \mathrm{n}$. Experiments on PBX (6) show evidence that global energy confinement can be improved with an applied limiter bias.

With these observations, a natural question arises: How might an electrical bias be best applied to a SOL so as to effect the global energy confinement characteristics of a tokamak (induce an 'artificial H-mode')? Or less ambitiously, one might simply ask: How might one design an effective bias scheme to actively control 
plasma fluxes and recycling in the boundary plasma, reducing the asymmetries that exist there?

\subsection{Model for Cross-Field Plasma Transport in a Biased Scrape-off Layer}

In order to address these questions, one must first develop a more quantitative model for cross-field plasma transport in a biased tokamak scrape-off layer. Consider a simplified model of a biased tokamak scrape-off layer (BSOL) shown in fig. 1. The BSOL is bounded along freld lines running parallel to the $z$ axis by limiters or divertor neutralizer plates spaced at a distance of $2 \mathrm{~L}$. The interface between the main plasma and BSOL occurs on the $z$ axis at $r=a$ and it is assumed that no gradients exist in the Bxf direction. The limiters are held fixed at some potential, $\Phi W$, which may be different than the local floating potential, $\Phi f$.

The BSOL is populated by a cross-field plasma flux $\left(\Gamma_{\perp}=\Gamma_{r}\right)$ from the main plasma region and depleted by a parallel plasma flux $\left(\Gamma_{/ /}=\Gamma_{Z}\right)$ flowing to limiter/divertor surfaces. For simplicity, neutral densities in the BSOL are assumed to be low enough so that ionization can be neglected in the particle balance. In an analogous manner, a cross-field plasma current $\left(J_{\perp}\right)$ is assumed to flow from the main plasma into the BSOL while a parallel plasma current $\left(\mathrm{J}_{/ /}\right)$flows to limiter surfaces (independent of the equilibrium plasma currents in the central plasma).

\section{A. Radial Fluxes and Currents}

Radial transport of electrons and ions can be modelled by

$$
\begin{aligned}
& \Gamma_{r}^{\theta}=-D_{\perp}^{e} \nabla_{r} n-\mu_{\perp}^{e} n E_{r} \\
& \Gamma_{r}^{i}=-D_{\perp}^{i} \nabla_{r} n+\mu_{\perp}^{i} n E_{r}
\end{aligned}
$$

where $D_{\perp}$ is typically on the order of Bohm and assumed to be governed by plasma turbulence. If the turbulence is due to low frequency electrostatic fluctuations, one expects that $D_{\perp}$ would be approximately equal for ions and electrons while $\mu_{\perp}$ might retain its classical value for each species. This is because electrostatic turbulence moves electrons and ions across the magnetic field through ExB motion at essentially the same rate. In any event, this analysis can be easily generalized to inclide anomalous values of all coefficients.

From eqs. 3 and 4, the radial plasma flux and current are 


$$
\begin{aligned}
& \Gamma_{r}=-D_{\perp}^{i} \nabla_{r} n+\mu_{.}^{\prime} n E_{r} \\
& \frac{J_{r}}{q}=-\left(D_{\perp}^{\prime}-D_{\perp}^{\theta}\right) \nabla_{r} n+\left(\mu_{\perp}^{i}+\mu_{\perp}^{\theta}\right) n E_{r} .
\end{aligned}
$$

In steady state, with no particle sources in the BSOL,

$$
\begin{aligned}
& \nabla_{\mathrm{r}} \cdot \Gamma_{\mathrm{r}}=-\nabla_{/ /} \cdot \Gamma_{/ /} \\
& \nabla_{\mathrm{r}} \cdot \mathbf{J}_{\mathrm{r}}=-\nabla_{/ /} \cdot \mathrm{J}_{/ /}
\end{aligned}
$$

and averaging eqs. (7) and (8) over $z$ from $z=0$ io $z=L$ yields

$$
\begin{aligned}
& \nabla_{r} \cdot \overline{\Gamma_{r}}=\cdot \frac{\Gamma_{\prime \prime}^{w}}{\mathrm{~L}} \\
& \nabla_{r} \cdot \overline{J_{r}}=-\frac{J^{\prime \prime}}{\mathrm{qL}}
\end{aligned}
$$

where $\Gamma_{\|}^{w}$ and $J_{\|}^{w}$ are the parallel flux and current at the limiter surface. Averaging eqs. (5) and (6) from $Z=0$ to $Z=L$ and using (9) and (10) yields

$$
\begin{gathered}
-D_{\perp}^{i} \nabla_{r}^{2} n+\mu_{\perp}^{i} \nabla_{r} \cdot n E_{r}=-\frac{\Gamma_{\| l}^{w}}{L} \\
-\left(D_{\perp}^{i}-D_{\perp}^{9} \nabla_{r}^{2} n+\left(\mu_{\perp}^{i}+\mu_{\perp}^{\theta}\right) \nabla_{r} \cdot n E_{r}=-\frac{J_{l l}^{w}}{q L} .\right.
\end{gathered}
$$

In this model, the transport coefficients are assumed to be independent of space and of $E_{1}$. The overbar notation that indicated an averaging operation along $Z$ in eqs. (9) and $(10)$ has now been dropped. Eliminating the $E_{r}$ terms by combining (11) and (12) results in 


$$
\left(D_{\perp}^{i} \mu_{\perp}^{\theta}+D_{\perp}^{\theta} \mu_{\perp}^{i}\right) \nabla_{r}^{2} n=\left(\mu_{\perp}^{i}+\mu_{\perp}^{g}\right) \frac{\Gamma_{l \prime}^{w}}{L}-\mu_{\perp}^{i} \frac{J^{w}}{q^{\prime}}
$$

and similarly eliminating $\nabla_{p}^{2} n$ terms in (11) and (12) yields

$$
\left(D_{\perp}^{i} \mu_{\perp}^{\theta}+D_{\perp}^{\theta} \mu_{\perp}^{i}\right) \nabla_{r} \cdot n \nabla_{r} \Phi=\left(D_{\perp}^{\theta}-D_{\perp}^{i}\right) \frac{\Gamma_{\mu}^{w}}{L}+D_{\perp}^{i} \frac{J^{w}}{q L}
$$

where $E_{r}=-\nabla_{r} \Phi$.

\section{B. Parallel Fluxes and Currents}

Along the magnetic field, presheath eleciric fields draw ions to the limiter surfaces satisfying the Bohm condition. This situation persists under a wide range of limiter biases as long as the local limiter potential remains approximately $0.5 \mathrm{Te}$ below the plasma potential at $z=0$. Thus the parallel plasma flux to the wall can be modelled as

$$
\Gamma_{/ l}^{w}=\alpha \cap c_{s}
$$

where $\alpha$ is a parameter in the range $0.5<a<1$, ailo $C_{S}$ is the ion sound speed.

The parallel plasma cuirent to the wall can be estimated from planar Langmuir probe theory. Given the same restriction on wall potential mentioned above,

$$
J_{1 \prime}^{w}=\alpha q n C_{s}\left[1-\exp \left(\frac{\Phi_{w}-\Phi_{f}}{T_{\theta}}\right)\right]
$$

By defining the plasma potential at the sheath eige, $\bar{\Phi}_{\mathbf{S}}$, to be zero, eq. (16) approximately applies for $-\infty<\Phi_{W}<0$. (Note that $\Phi_{f}$ is a negative quantity.) 


\section{BSOL Density and Potential Profiles}

Combining (15) and (16) with (13) and (14) results in an equation for the density profile in the BSOL,

$$
L\left(D_{\perp}^{\prime} \mu_{\perp}^{\theta}+D_{\perp}^{\theta} \mu_{\perp}^{i}\right) \nabla_{r}^{2} n=\alpha n C_{s}\left[\mu_{\perp}^{\theta}+\mu_{J}^{\prime} \exp \left(\frac{\Phi_{W}-D_{f}}{T_{\theta}}\right)\right] \text {, }
$$

and an equation for the potential profile,

$$
L\left(D_{\perp}^{i} \mu_{\perp}^{\theta}+D_{\perp}^{e} \mu_{\perp}^{i}\right) \nabla_{r} \cdot n \nabla_{r} \Phi=\alpha n C_{S}\left[D_{\perp}^{e}-D_{\perp}^{i} \exp \left(\frac{\Phi_{W}-\Phi_{f}}{T_{\theta}}\right)\right] \text {, }
$$

for a prescribed wall potential profile. Nore that $\Phi_{f}$ is coupled to $\Phi$ (throtgh $T_{e}$ for example).

Undor the condition that the limiter surface is biased in such a way as to hold $\left(\Phi_{W}-\Phi_{f}\right) / T_{\theta}$ constant in $r$ (by properly specifying $\Phi_{W}$ and/or $T_{\theta}$ profiles), the density profile that solves (17) and meets the bounday condition of zero density as $r$ $\rightarrow \infty$ is simply

$$
n(r)=n_{0} \exp (-r / \lambda)
$$

where

and

$$
\begin{aligned}
& D^{\circ}=D f\left(\Phi_{w}\right) \\
& \lambda=\sqrt{\frac{D^{\prime} L}{\alpha C_{s}}}
\end{aligned}
$$

$$
f\left(\Phi_{w}\right)=\frac{\mu_{\perp}^{\theta}+\frac{i}{\mu_{\perp}^{e}+\mu_{\perp}^{i} \exp \left(\frac{\Phi_{w}-\Phi_{H}}{T_{\theta}}\right)}}{\mu^{\prime}}
$$

and it has been assumed that $D_{\perp}^{\theta}=D_{\perp}^{i}=D$. 
In the case of a non-conducting or locally floating limiter, eqs. (19)-(22) yield $f\left(\Phi_{w}\right)=1, D^{*}=D$, and the density profile has an e-folding length that is related to $D$ through the widely accepted formula

$$
D=\frac{\alpha \lambda_{0}^{2} C_{S}}{L}
$$

For later reference, the scrape-off length for this case of a locally floating limiter is denoted by $\lambda_{0}$.

Similarly, the potential profile that solves (18) and remains bounded as $r \rightarrow$ $\infty$ is

$$
\Phi(r)=-\frac{D^{*}\left[1-\exp \left(\frac{\Phi_{w}-\Phi_{t}}{T_{\theta}}\right)\right] r}{\left(\mu_{\perp}^{\theta}+\dot{H}\right) \lambda}+\Phi_{0}
$$

The above analysis applies only to the case when the quantity $\left(\Phi_{W}-\Phi_{f}\right) / T_{\theta}$ is independent of $r$. This is actually quite restrictive. In general, a biased scrape-off layer will have radial potential and temperature profiles that are completely decoupled. A metallic limiter, even though it is not actively biased, will effectively impose a positive bias at some radial locations and a negative bias at other radial locations. Nevertheless, within the restrictions implicit in eqs. (19)-(24), one can make some simple quantitative estimates of the effect of an applied bias on density profiles in a BSOL.

Equations (19)-(22) predict that the density e-folding length will increase for negative limiter biases and decrease for positive limiter biases (relative to the local floating potential). It is important to note that this model predicts a significant modification to the density e-folding length, independent of the magnitudes of $\mu_{\perp}^{e}$ and $\mu_{\perp}^{i}$ relative to $D$. Since no explicit relationship (e.g. Einstein relation) was postulated between the phenomenological mobility and diffusion coefficients in deriving eqs. (19)-(24) an interesting situation arises: Although the value of $D$ determines the overall scale of the density e-folding length, even a siight amount of crosi-field mobility (classical mobility from neutral collisions, for example) may allow one to greatly modify the density e-folding length through an electrical bias. Given that the edge plasma is strongly turbulent, it is difficult to estimate the appropriate phe:iomenological values for $\mu_{\perp}^{e}$ and $\mu_{\perp}^{i}$. Nevertheless, consider the following three scenarios: (1) $\mu_{\perp}^{j} \gg \mu_{\perp}^{e}$, (2) $\mu_{\perp}^{i}=\mu_{\perp}^{\theta}$, and (3) $\mu_{\perp}^{i} \ll \mu_{\perp}^{\theta}$. 


\section{Case 1: $\mu_{\perp}^{i} \gg \mu_{\perp}^{\theta}$}

if one assumes that the electron and ion mobilities are classical and determined by neutral collisions, then $\mu_{\perp}^{\prime} / \mu_{\perp}^{\theta} \sim m_{i} / m_{e}$ when the ion-neutral and electron-neutral collision frequencies are comparable. Even accounting for typical values of ionneutral and electron neutral collision cross-sections in the SOL plasma one may more accurately estimate that $\mu_{\perp}^{i} / \mu_{\perp}^{\theta} \sim 0.2 \mathrm{~m} / \mathrm{m}_{\theta}$ (hydrogen plasma). In any event, $\mu_{\perp}^{\theta}$ can be dropped relative to $\mu_{\perp}^{i}$ (as long as $\Phi_{w}-\Phi_{1} \geq-T_{\theta} \ln \left[\mu_{1}^{i} / \mu_{\perp}^{\theta}\right]$ ) and eq. (22) becomes

$$
f\left(\Phi_{w}\right)=\exp \left(\frac{\Phi_{\mathbf{Y}}-\Phi_{w}}{T_{\theta}}\right)
$$

Consequently, the density e-folding length due to an applied bias is simply

$$
\lambda=\exp \left(\frac{\Phi_{i}-\Phi_{w}}{2 T_{\theta}}\right) \lambda_{0}
$$

This case vields the most dramatic change in density scrape-off length for a given bias. Modified e-folding lengths in the range $0.17<\lambda \lambda_{0}<14.0$ are attainable for plasma w:th $\Phi_{t}=-3.5 T_{e}$ and $\Phi_{w} \leq 0$.

Case 2: $\mu_{\perp}^{i}=\mu_{\perp}^{\Theta}$

It is possible to imagine situations where the perpendicular electron mobility is strongly enhanced by parallel electron transport through electromagnetic turbulence or by an ergotic magnetic field structure in the SOL plasma. If $\mu_{1}^{\theta}$ becomes comparable to $\mu_{1}^{i}$, the dcasity e-folding length is,

$$
\lambda=\lambda_{0} \sqrt{\frac{2}{1+\exp \left(\frac{\Phi_{w}-\Phi_{H}}{T_{\theta}}\right)}} .
$$

The scrape-off length modification accessible to the BSOL is then $0.24<\lambda / \lambda_{0}<1.4$ for plasma with $\Phi_{\uparrow} \approx-3.5 T_{e}$ and $\Phi_{W} \leq 0$. 


\section{Case 3: $\mu_{\perp}^{i} \ll \mu_{\perp}^{\theta}$}

In this extreme limit, limiter bias has no effect on the density profile and the efolding length is fixed at $\lambda=\lambda_{0}$.

\subsection{Comparison of Model with Results from the ALT-I Bias Experiments in TEXTOR}

Experiments in tokamak scrape-off layers indicate a dependence of the SOL density e-folding length on limiter bias which is qualitatively similar to the dependences outlined in eqs. (26) and (27). In TEXTOR, the ALT-I pump limiter was used as the primary limiter and biased with respect to the chamber wall and secondary limiters at larger minor radii [7]. This set of experiments was aimed at testing the plasma response to a radial potential drop imposed between the wall and the last closed flux surface.

Figure 2, from Ref. [7], shows radial density profiles in the SOL for the case of three limiter bias conditions: (1) ALT-I 'floating' with respect to the walls and other limiters; (2) ALT-I biased negative at -450 volts; and (3) ALT-I biased positive at +450 volts. As suggesied by the BSOL model developed above, the ALT-I limiter bias arrangement and subsequent profile modification might be explained by a two zone radial BSOL model as shown in fig. 3. Plasma in biased s rape-off layer zone A (BSOL-A) is depleted along field lines by ALT-I while BSOL-B is connected along field lines to other limiters and wall structures ir TEXTOR. Equations (26) and (27) predict that a negative bias applied to the limiters in zone A (ALT-I) with respect to zone $B$ (other limiters and wall) should result in an increase in the density efolding length in zone A with respect to zone B. This is qualitatively observed in fig. 2. Results from positive ALT-I bias also suggest a shortening of the scrape-off length in zone $A$ and a lengthening of scrape-off length in zone $B$, consistent with the model. Refinements to this simplified BSOL model might allow one to more accurately predict the detail in the radial density profile structure. Such refinements may include particle sources due to ionization, radial temperature and potential profiles, transport coefficients that depend on space, and a proper matching of density and density gradients between bias zones. Nevertheless, the essential dependence of the SOL density e-folding length on a biased limiter surface appears to be contained in the simple model outlined above.

The most dramatic finding in the bias experiments on TEXTOR was that the ALT-I pump limiter particle removal efficiency could be almost doubled by a negative ALT-I bias [7]. Thus it is clear that ion fluxes to limiters and wall surfaces can be controlled by an applied electrical bias in the edge plasma. This suggests that 
other innovative approaches may exist for edge particle and recycling control, including the concept of an $m=1$ Electrostatic Barrier Scrape-off Layer.

\section{AN $m=1$ ELECTROSTATIC BARRIER SCRAPE-OFF LAYER (EBSOL)}

\section{1 $m=1$ versus $m=0$ Biased Limiter Structures}

All bias experiments performed in tokamaks to date can be broadly classified as having an $\mathrm{m}=0$ biased scrape-off layer, or BSOL structure, as shown in fig. 4 (a). Here, the ' $m$ ' number corresponds to the poloidal wavenumber as it is usually defined for magnetic perturbations in tokamaks. The simplest biased limiter geometry has $m=0$ since it involves inserting a single limiter into the discharge and biasing it with respect to the wall (or injecting electrons $[3,4]$ ). To a first approximation, there exists no poloidal variation in the applied potential and the plasma may be coaxed to preferentially scrape-off either on the inserted limiter or on the wall, depending on limiter bias polarity. Conceptually, a radial electrostatic barrier may be formed between the inserted limiter and wall structures. Although it is typically observed that the global particle confinement time can be changed, some side effects include impurity accumulation in the central discharge and/or increased sources of impurities at the edge.

In contrast to this usual scenario, we propose the $m=1$ Electrostatic Barrier Scrape-Off Layer scheme ( $m=1$ EBSOL) as shown in figs. 4 (b) and (c). The SOL is divided poloidally into two zones, both exhibiting top-bottom symmetry: one on the larger major radius edge of the torus and one on the smaller major radius edge. Magnetic field lines which would normally connect these two zones by the rotational transform are intercepted by pairs of limiters or divertor plates positioned at the boundary between the zones. These pairs of plates are biased so that the outer SOL acquires a different potential relative to the inner SOL, as shown. The primary goal is to change the cross-field plasma transport in the outer BSOL relative to the inner BSOL such as through effects outlined in section 2.2.

On first inspection, it may not $b c$ obvious why the $m=1$ scenario is desirable or beneficial for the operation of the tokamak. In a tokamak plasma that normally exhibits poloidal symmetry, this scenario would appear to simply change the poloidal location that the plasma chooses to exhaust into the scrape-off layer layer. One would expect that the total rate of plasma loss would remain approximately unchanged. However, it is now widely recognized that most, if not all tokamaks exhibit strong poloidal asymmetries in cross-field plasma transport, favoring a higher transport rate on the larger major radius edge of the torus. It is also known that the radial electric field in the plasma edge plays an important role in plasma transport due to electrostatic fluctuations [5] (or within the context of the model developed in section 2.2, cross-field diffusion is a strong function of radial electric field). Thus we conjecture that an $m=1$ EBSOL scheme could be used to 'stopper up' the high 
field transport on the large major radius side and force plasma to exhaust instead on the small major radius side of the torus. In any case, even a simple redistribution of fluxes in the SOL from such an $m=1$ scheme may be desirable from the point of view of controlling plasma and heat fluxes to limiter or divertor plates.

\subsection{Observations of Perpendicular Transport Asymmetries}

There is both direct and indirect evidence of perpendicular transport asymmetries in the particle and heat fluxes that originate from the central plasma of a tokamak discharge. Data taken from probes in the scrape-off layer plasma of ALCATOR C directly showed that the cross field particle transport was higher on the large major radius edge than on the small major radius edge of this toroidal plasrna [8]. Similar inside/outside asymmetries in cross field particle and heat fluxes have been detected in other tokamaks by more indirect means, including the observation that plasma tends to unload onto outer collector plates in the diverted discharges of ASDEX and PDX [9]. The existence of a cooler, radiating, 'MARFE' plasma on the small major radius edge of most tokamaks $[11,12]$ is further evidence for an preferentially lower cross-field energy transport rate into that poloidal sector of the edge plasma. It has been speculated that the increased transport on the outside of the torus results from ballooning modes [13,14]. In $\mathrm{H}$-mode discharges, edge localized modes (ELMS) appear to be concentrated on the outside midplane of the torus [15]. Recent probe measurements of density and potential fluctuations in the CCT tokamak at UCLA show significantly higher fluctuation levels on the outer versus the inner major radius edge of the torus during ohmic discharges [16].

\section{3 nin=1 EBSOL Voltages and Currents}

Assume that one considers building an $m=1$ EBSOL biasing scheme for a tokamak. What are the voltages and currents that would be required to 'equalize' the inside-outside transport asymmetry intrinsic to a tokamak? Of course, the exact answer to this question requires a model that can handle the dependence of turbulent cross-field transport on the applied radial electric field. Nevertheless, the model developed in section 2.2, which assumes that $D$ and $\mu$ do not depend on $E_{\mathrm{F}}$, can provide a crude estimate of the required voltages and currents. These estimates may be viewed as 'conservative' in that in accounting for $D\left(E_{r}\right)$ and $\mu\left(E_{r}\right)$, the required bias voltages may be significantly reduced.

\section{A. Bias Voltages for $m=1$ EBSOL: Matching of Inside and Outside Currents}

Referring to fig. 4, one sees that the electrical circuit for the $m=1$ EBSOL is the same as for a very large double probe, with one electrode being the outer divertor or limiter plates and the other electrode being the inner set of plates. However, the 
effective 'wetted areas' of these electrode $;$ is characterized by the density e-folding length rather than the electrode size. Del:ote the density e-folding length on the outside as $\lambda^{\text {out }}$ and on the inside as $\lambda^{\text {in }}$. The total currents to the inner and cuter plates can be estimated by integrating eq. (16) in minor radius yielding

$$
\begin{aligned}
& I^{\text {out }}=\alpha 4 \pi q n_{0} C_{s} \lambda^{\text {out }} R_{0} \sin \psi\left[1-X^{2}\right] \\
& I^{\text {in }}=\alpha 4 \pi q n_{0} C_{s} \lambda^{\text {in }} R_{0} \sin \psi\left[1-Y^{2}\right]
\end{aligned}
$$

where $n_{0}$ is the density at the last closed flux surface (assumed to be independent of polcidal angle), $\psi$ is the angle that magnetic field lines in the SOL intercept the collector plates, $R_{0}$ is the major radius, and $q$ is the electron charge. The following definitions have been employed in writing (28) and (29):

$$
\begin{aligned}
& X=\exp \left(\frac{\Phi_{w}^{\text {out }}-\Phi_{f}}{2 T_{\theta}}\right) \\
& Y=\exp \left(\frac{\Phi_{f}^{\text {in }}-\Phi_{f}}{2 T_{\theta}}\right) .
\end{aligned}
$$

Similar to the analysis in section 2.2 , it has been assumed that $X$ and $Y$ are constant in minor radius. As discussed earlier, this somewhat restrictive assumption has been invoked only to simplify the analysis. For the case of limiter/divertor plates biased to spatially uniform potentials of $\Phi_{w}^{\text {in }}$ and $\Phi^{\mathrm{O}} \mathrm{w}$, , one would need to 'adjust' the $T_{e}$ profiles so that the quantities $\left(\Phi_{w}\right.$ - $\left.\Phi_{f}\right) / T_{e}$ and $\left(\Phi{ }_{w}\right.$ out $\left.-\Phi_{f}\right) / T_{e}$ remain constant in space. Of course, a more careful analysis might fix the $T_{\theta}$ profile and compute the self-consistent density and potential profiles through simultaneous solutions of eqs. (13) and (14). However, the presentation of such an involved analysis would only serve to detract from the main points made in this discussion.

The difference in wall potentials is fixed by the voltage on the external power supply, $\Delta V=\Phi_{w}^{\text {out }}-\Phi_{w}^{\text {in }}$. Defining the quantity

$$
\rho=\exp \left(\frac{\Delta V}{2 T_{e}}\right)
$$

one may simply write that

$$
X=p Y .
$$


A closed electrical circuit requires that the sum of equations (28) and (29) be equal to zero. Modelling the dependence of the e-folding length on electrical bias as eq. (26), this condition can be written

$$
\frac{\lambda_{0}^{\text {out }}}{X}\left[1-X^{2}\right]+\frac{\lambda_{0}^{\text {in }}}{Y}\left[1-Y^{2}\right]=0
$$

where $\lambda_{0}^{\text {out }}$ and $\lambda_{0}^{\text {in }}$ are the scrape-off lengths for the case of electrically nonconducting collector plates. Solving for $X$ in terms of $\eta_{0}\left(=\lambda_{0}^{i n} / \lambda^{\circ}{ }_{0}^{\mathrm{t}}\right)$ and $\rho$ yields

$$
X=\rho \sqrt{\frac{\eta_{0}+1 / \rho}{\eta_{0}+\rho}}
$$

so that the wall potentials, $\Phi_{\mathrm{W}}^{\text {Out }}$ and $\Phi_{W}^{\mathrm{in}}$, for a given power supply bias, $\Delta \mathrm{V}$, can be evaluated through (30)-(35). The modified density e-folding lengths are explicitly

$$
\begin{aligned}
& \lambda^{\text {out }}=\frac{\lambda_{0}^{\text {out }}}{\rho} \sqrt{\frac{\eta_{0}+\rho}{\eta_{0}+1 / \rho}} \\
& \lambda^{\text {in }}=\lambda_{0}^{\text {in }} \sqrt{\frac{\eta_{0}+\rho}{\eta_{0}+1 / \rho}}
\end{aligned}
$$

and most importantly, the new ratio of e-folding lengths due to applied bias is

$$
\frac{\lambda^{\text {in }}}{\lambda^{\text {out }}}=\frac{\lambda_{0}^{i n}}{\lambda_{0}^{\text {out }}} \exp \left(\frac{\Delta V}{2 T_{\theta}}\right)
$$

This is the major result. The model predicts that a factor of two change in the inside/outside scrape-off length ratio is possible with a relatively weak bias of $\Delta V$ $=1.4$ times $T_{e}\left(\Phi_{w}\right.$ at both walls must satisfy $\Phi_{w} \geq \Phi_{\uparrow}-T_{e} \ln \left[\mu_{\perp}^{i} / \mu_{\perp}^{\theta}\right]$ in order for eqn. (38) to be a valid approximation). In the case $\eta_{0}=1$, a power supply voltage, $\Delta V$, results in a change in the plasma-wall sheath drops of $\pm \Delta V / 2$ at the outer and inner $F$ lates, respectively 
Measurements of $\lambda_{0}^{\text {out }}$ and $\lambda_{0}^{\text {in }}$ on the Alcator $C$ tokamak [8] suggest that $3<$ $\lambda_{0}^{\text {out }} / \lambda^{i} 0^{n}<8$. This implies that the power supply voltage for an $m=1$ EBSOL required to 'equalize' the transport in a tokamak is roughly

$$
\Delta V \leq 3 \times T_{\theta}
$$

leading to a change in plasma-wall sheath drops of less than $\pm 1.5 \mathrm{Te}$ at the outer and inner plates. It is important to note that this model computes a transport modification due entirely to cross-field mobility. Any increase or decrease in cross-field diffusion through changes in electrostatic turbulence has been neglected. In this respect, eq. (39) is conservative and may be viewed as an upper bound on the required $\Delta V$.

B. Bias Currents for $m=1$ EBSOL: Plasma Fluxes into the SOL

Consider a circular cross-section tokamak with major radius, $R_{0}$, and minor radius, a. The global particle confinement time, $\tau_{p}$, may be expressed as

(40)

$$
\tau_{p}=\frac{\int_{v o l} n \delta V}{\int_{\text {surf }} \Gamma \cdot \delta A} .
$$

The surface integral is typically computed over the last closed magnetic flux surface. For the case when ionization is not significant within the scrape-off plasma. the denominator of (40) also corresponds to the total plasma flux on limiters, divertor plates and wall surfaces. Modelling the central plasma density profile as a parabola in minor radius to some power, $\gamma$, the total plasma efflux across the last closed flux surface (written as an equivalent current, $I^{*}$ ) may be approximated as

$$
I^{w}=\frac{2 q \pi R_{0} a^{2} n_{0}}{\tau_{p}(x+1)}
$$

where $n_{0}$ is the plasma density on axis and $q$ is the electric charge. The required power supply current for an $m=1$ EBSOL would need to be on the order of $I^{w}$ since a significant fraction of $\mathrm{I}^{\mathrm{w}}$ would be redistributed on limiter/divertor plates. 
It is interesting to note that in a confinement regime where $\tau_{p}$ is proportional to the energy confinement time, $\tau_{\theta}$, and $\tau_{\theta}$ scales according to the neo-Alcator law,

$$
\tau_{p} \propto \tau_{\theta} \propto n_{0} R_{0}^{2} a \text {, }
$$

then eq. (41) yields for the dependence of $I^{w}$,

$$
I^{W} \propto \frac{a}{R_{0}(\gamma+1)}
$$

This tells us that the current to the walls is quite insensitive to the size and density of the tokamak plasma. Thus, as a tokamak becomes larger, the power expended on the $m=1$ EBSOL remains nearly constant. The ratio of the $m=1$ EBSOL power to the total power used in maintaining the discharge becomes smaller.

\subsection{The $m=1$ EBSOL as an ExB Divertor}

The $m=1$ EBSOL concept not only imposes an electrostatic barrier to particles crossing into the SOL but also may efficiently sweep particles to the limiter/divertor plates through an ExB drift. The result is similar to the 'ExB scraper' concept investigated by Strait et al. [1,2] which used biased wall surfaces aligned parallel to B. $H$ vever, unlike the ExB scraper experiment, the $m=1$ EBSOL limiter/divertor plates can collect large currents along field lines, and therefore maintain large crossfield plasma potential perturbations throughout the SOL volume.

Figure 5 illustrates the cross-field flow patterns for: (a) an ExB divertor in a limiter corfiguration and (b) an ExB-assisted divertor configuration respectively. Within the scrape-off layer, applied electric fields cause a vertical ExB drift of plasma towards the upper pair of limiter/divertor plates. The impact of the ExB drift becomes important when its magnitude approaches the poloidal component of the parallel plasma flow velocity (reduced sound speed) to the limiter/divertor plates, i.e. when

$$
\frac{\left|E_{r}\right|}{B}=\frac{C_{s} B_{\theta}}{B}
$$

Defining a dimensionless 'figure of merit' as 


$$
\gamma=\frac{\left|E_{r}\right|}{C_{s} B_{\theta}}
$$

the Exi drift is important when $\gamma$ approaches 1 .

The magnitude of the perpendicular electric field can be estimated from the modei developed in section 2.2. In the limit when $\mu_{\perp}^{i} \gg \mu_{\perp}^{9}$, eq. (24) can be written as

$$
\Phi(r)=\frac{2 D r}{\mu_{\perp}^{i} \lambda_{0}} \sinh \left(\frac{\Phi_{w}-\Phi_{T}}{2 T_{\theta}}\right)+\Phi_{0}
$$

so that approximately

$$
\left|E_{r}\right|=\frac{D}{\mu_{\perp} \lambda_{0}}
$$

when the wall bias is taken to be $\Phi_{W} \approx \Phi_{Y} \pm T_{\theta}$. This result is consistent with that inferred from eq. (1) under the condition that an 'electrostatic barrier' forms.

Note that the magnitude of the perpendicular electric field depends on the ratio of cross-field diffusion over ion mobility (in this limit when $\mu_{\perp}^{i} \gg \mu_{\perp}^{\theta}$ ). This is in direct contrast to the modification of the density e-folding length (see eqs. (19)-(22), for example) which depends only on the relative magnitudes of cross-field ion and electron mobilities. Since the appropriate magnitude of $\mu_{\perp}^{i}$ is not known for the turbulent edge plasma, a reliable estimate of $\left|E_{r}\right|$ is difficult to obtain. Some assumption about the relative magnitude of $D$ and $\mu_{1}^{i}$ must be made.

Defining the dimensioniess quantity,

$$
\beta=\frac{D}{\mu_{\perp}^{i} T_{\theta}} \text {, }
$$

the parameter $\gamma$ becomes

$$
\gamma=\frac{\beta T_{e}}{\lambda_{0} C_{s} B_{\theta}}
$$


Consider the following two extreme cases (still satisfying $\mu_{\perp}^{i} \gg \mu_{\perp}^{\theta}$ ): (1) diffusion and molility obey the Einstein relation; and (2) diffusion is anomalous (Bohm) but the ion mobility is classical and govemed by ion-neutral collisions.

Case 1: Diffusion and Mobility obey the Einstein Relation

In this case, $\beta \approx 1$ (assuming $T_{i}-T_{\theta}$ ), and for a deuterium SOL plasma with $T_{i}=T_{\theta}=25 \mathrm{eV}, B_{9}=0.5$ tesla, and $\lambda_{0}=0.01 \mathrm{~m}$,

$$
\gamma=0.1
$$

ExB drifts therefore play only a minor rele in this scenario.

Case 2: Bohm Diffusion and Classical Ion Mobility

In this case,

$$
\beta=\frac{\mu_{/ 1}^{i} B}{16} \text {, }
$$

where perpendicular ion mobility has been replaced by parallel ion mobility through the relationship $\mu_{1}^{i} \neq 1 /\left(\mu_{1}^{i}, B^{2}\right)$. Parallel ion mobility is governed primarily by charge exchange collisions and scales inversely with the charge exchange rate. For a neutral density of $10^{12} / \mathrm{cm}^{3}$ and a toroidal magnetic field of 5 tesla, $\beta \approx 10^{3}$, so that from eq. (49),

$$
\gamma \approx 10^{2}
$$

Thus, ExB drifts in this scenario play an importent role. However, it is not clear that the flow can actually exceed the reduced sound speed $(\gamma=1)$ without driving strong turbulence. Shocks are theoretically predicted to occur at this 'resonance' flow speed and can greatly increase the cross-field transport [17]. It may be more realistic to assume that once $\gamma$ approaches 1 , strong plasma turbulence modifies the effective mobility and diffusion coefficients such that the electric field is reduced and $\gamma$ cannot exceed 1.

These two cases clearly demonstrate two important points: (1) the impact of ExB drifts in the $m=1$ EBSOL uitimately depends on the magnitude of cross-field 
mobility relative to cross-field diffusion; and (2) except in the case when cross-field ion mobility is anomalously high and comparable to cross-field diffusion (through the Einstein relation), ExB drifts in the $m=1$ EBSOL may supplement or even replace a magnetic divertor system for plasma exhaust and active SOL plasma flux control.

It is known that ExB flows can be more effective than parallel plasma flows for removing plasma and impurities in the boundary plasma [2]. For example, impurities must be accelerated to the bulk plasma flow speed along field lines by frictional drag in order to be removed in a magnetic divertor. In contrast, bulk plasma and impurities are removed with equal efficiency by EXB flcws.

\subsection{Refinements to the EBSOL Scheme: High Recycling Divertor, Other Biases}

The BSOL model in section 2.2 assumes that the plasma density and temperature at the limiter/divertor plate maps along field lines to zones of comparable plasma densities and temperatures in the SOL. However, contrary to this assumption, a ligh recycling divertor will maintain density and temperature gradients along magnetic field lines. The high density, low temperature plasma in a high recycling divertor may significantly reduce the required electrical bias in an $m=1$ EBSOL scheme. Since electron heat fluxes and ion sputtering rates depend strongly on the plasma-wall sheath potential drop, the capability to reduce the bias voltage while maintaining the SOL transport modification is advantageous.

Equations (13)-(16) illustrate how this reduction in required bias voltage occurs. Fluxes $\left(\Gamma_{/ /}^{w}\right)$ and currents $\left(\mathrm{J}_{/ / 1}^{w}\right)$ on the right hand sides of eqs. (13) and (14) are determined by the plasma conditions at the limiter/divertor plate and the bias of the plate relative to the local floating potential. The impact of an electric bias on the perpendicular density and potential profiles simply depends on the local current density at the plates which is modelled by eqn. (16) using planar Langmuir probe theory.

Consider a high recycling divertor $(H)$ and a low recycling divertor $(L)$ that have the same electron pressures at the divertor plate, $n^{H} \times T_{\theta}^{H}=n^{L} \times T_{e}^{L}$. The ratio of densities in these two regimes may be written as $\beta=n^{\mathrm{H}} / n^{\mathrm{L}}$. Since the net current to the divertor plate (I) scales approximately as

$$
I \propto n \sqrt{T_{\theta}}\left[1-\exp \left(V_{b} / T_{\theta}\right)\right]
$$

for a given plate bias about the floating potential $\left(V_{b}\right)$, it can be shown that the bias in the high recycling regime can be reduced by the fraction

$$
v_{b}^{H} / V_{b}^{L} \approx \beta^{-3 / 2}
$$


for $V_{b}^{L} \leq T_{\theta}^{L}$.

The concept of forming an 'electrostatic barrier' may also be used in other bias geometries. Figure 6 illustrates two bias schemes that may be emplo, red in conjunction with a divertor-based $m=1$ EBSOL: (i) divertor throat bias, and (ii) 2 bias of the plasma facing wall. Since the divertor plates are in good electrici: contact with the plasma, the divertor throat and/or the wall can be biased positive with respect to the plasma to reduce both the flux and energy of ions striking these surfaces. The effect of biasing the wall positive is similar to the $m=0$ EBSOL concept discussed in section 3.1, forcing the plasma to recycle more $\mathrm{c} n$ the limiter/diverto: plates and less on the wall.

\subsection{Effect of 'Intrinsic Bias' on Transport Analysis and Transport Asymmetries}

In the usual treatment of transport in a SOL, it is assumed that the crnss-field plasma flux is modeled by a diffusive pmcess. In the case of negligible ionization in the scrape-off layer, eq. (23) is typically used to relate the measured density efolding length to the effective diffusion coefficient. However, this formula is valid only in the restrictive case when local ambipolarity is satisfied, i.e. all wall surfaces that intercept the magnetic field lines in the SOL behave as nonconductors. Equations (19)-(22) therefore illustrate an important point: Although these relationships were developed from a simplified transport model, they clearly demonstrate that when wall surfaces are held at a potential different than the local floating potential, equation (23) no longer afplies and one must consider cross-field fluxes from mobility-like transport and ExB flows as well as from diffusive-like transport.

It is well established that a tokamak SOL is globally ambipolar but locally nonambipolar. Currents may flow into and out of metallic surfaces. Some types of poloidal transport asymmetries reported in tokamak scrape-off layer plasmas may therefore be caused not only from a spatial variation in the diffusive component of the flux but also from the spatial structure of the 'intrinsic bias' that exists on limiter, divertor plate, and wall surfaces.

\section{SUMMARY}

A new biased limiter concept is proposed, an $m=1$ Electrostatic Barrier Scrape-Off Layer ( $m=1$ EBSOL), which may allow an active contril of cross-field plasma flux into a tokamak scrape-off layer. The technique is motivated by experimental observations that particle losses in tokamak edge plasmas are poloidally asymmetric, favoring higher loss rates on the large major radius side of the torus. Conceptually, the $m=1$ EBSOL imposes an 'electrostatic barrier' to cross-field ion 
transport on the large major radius side, forcing plasma to exit on the small major radius side instead.

Measurements have shown that an externally applied radial electric field can significantly reduce the plasma transport due to electrostatic fluctuations [5]. Thus, this scrape-off layer 'biasing' scheme may provide a means to reduce the turbulencedriven transport in the edge plasma on the outside half of the torus. In the most optimistic light, one may even envision inducing an 'artificial H-mode', by extemally supplying the strong radial electric field that is believed tu piay an important role in $\mathrm{H}$-mode discharges [10].

A simple model for cross-field transport in a biased scrape-off layer is developed which, as a first approximation, ignores any dependence of the cross-field diffusion and mobility on the applied radial electric field. It is shown that although diffusion determines the overall scale of the density e-folding length, the ratio of ion to electron cross-field mobility solely determines the impact of an eiectrical bias on the density e-folding length. Hence, the quantitative relationship between diffusion and mobility need not be known. The model is found to qualitatively predict the overall scrape-off layer density profile modification seen in limiter bias experiments.

Voltages and currents required for the $m=1$ EBSOL scheme to 'equalize' the asymmetric transport into a tokamak SOL are estimated using the simple transport model. It is shown that voltages on the order of the SOL electron temperature and currents on the order of the central plasma refueling rate would be required. Power requirements for the $m=1$ EBSOL scheme are relatively insensitive to piasmd size and central plasma density in moderate density, ohmic heated tokamaks.

ExB flows induced by the $m=1$ EBSOL are considered. Large radial electric fields can be maintained in the SOL because of the good electrical contact of the $m=1$ EBSOL electrodes with the plasma along magnetic field lines. Unlike the density cfolding length, the magnitude of the potential gradient depends on the ratio of diffusion to mobility. Consequently, ExB drift velocities are model-dependent and difficult to estimate. Estimates of the ExB drift velocity for the case of classical ion mobility (ion-neutral collisions) and anomalous diffusion (Bohm) yield drift velocities greatly exceeding the ion sound speed. Recognizing that such flow velocities are nonphysical and result in the formation of instabilities and shocks, the EXB drift velocity is assumed to be bounded at the reduced sound speed, $V=C_{S}$ $\mathrm{B}_{0} / \mathrm{B}$. This is equivalent to assuming that the ratio of diffusion to ion mobility is determined by plasma turbulence and limits the allowed magnitude of the perpendicular electric field. In any event, such ExB drift velocities result in a plasma exhaust that is comparable to that in a poloidal divertor. The $m=1$ EBSOL may therefore be used to supplement or replace a magnetic divertor system for plasma exhaust and active SOL plasma flux control.

Recognizing that large voltages applied to limiter/divertor plates may result in enhanced electron heat fluxes or increased ion sputtering, the use of an $m=1$ EBSOL in a high recycling divertor is examined. It is argued that the high density and low 
temperature plasma in front of the divertor neutralizer plate may allow one to draw the required plasma currents at lower bias voltages. Bias voltages on the order of the divertor plate electron temperature would be required.

Other 'electrostatic barrier' biasing schemes are possible including a positive bias of the divertor throat and plasma facing wall relative to the divertor neutralizer plates. Such bias schemes combined with the $m=1$ EBSOL protect these surfaces from plasma ion sputtering while at the same time provide plasma exhaust and impurity control.

Finally, even in the absence of an applied bias, mobility-like transpor and ExB flows should be considered in the analysis of tokamak scrape-off layer plasmas. Transport due to such 'intrinsic biases' may play a role in the asymmetries that are in fact detected in tokamaks.

In summary, the $m=1$ Electrostatic Barrier Scrape-off Layer, or E3SOL, may be a jowerful tool for controlling fluxes in the tokamak boundary layer plasma and for improving the confinement characteristics of the central plasma. The utility of bias techniques such as the $m=1$ EBSOL is jifficult to assess theoretically, owing to the strong turbulence that exists in a scrape-off layer plasma. The next step in the logical development of the $m=1$ EBSOL concept and similar schemes is to investigate these ideas experimentally.

\section{ACKNOWLEDGEMENTS}

This work is supported by the U.S. Department of Energy.

\section{REFERENCES}

[1] Strait, E.J., Kerst, D.W., Sprott, J.C., 'Experimental Demonstration of ExB Plasma Divertor', Phys. Fluids 21 (1978) 2342.

[2] Strait, E.J., 'Poioidal Diveror Experiment with Applied ExB/B ${ }^{2}$ Drift',Nuci. Fusion 21 (1981) 943 .

[3] Taylor, R.J., James, B.W., Jin, S.X., Keller, L., LEE, P.S.C., Luhmann Jr., N.C., Morales, G.J., Oren, L., Park, H., Peebles, W.A., Talmadge, S., Yu, C.X., 'Particle Transport Due to ICRF Waves and Radial Electric Fields in Tokamaks', Proceedings of 9th IAEA Conference on Plasma Physics and Controlled Nuclear Fusion Research, Baltimore, Vol, III (1982) p. 251.

[4] Oren, L., Keller, F., Schwirzke, F., Talmadge, S., Taylor, R.J., 'Influence Exerted by the Plasma Edge Potential on Recycling. Sputtering and Impurity Accumulation', J. Nucl. Mater. 111\&112 (1982) 34. 
[5] Phillips, P.E., Wootton, A.J., Rowan, W.L., Ritz, Ch.P., Rhodes, T.L., Bengtson, R.D., Hodge, W.L., Durst, R.D., McCool, S.C., Richards, B., Gentle, K.W., Brower, D.L., Peebles, W.A., Luhmann Jr., N.C., Schooch, P., Forster, J.C., Hickok, R.L., Evans, T.E., 'Biased Limiter Experiments on TEXT, J. Nucl. Mater. 145-147 (1987) 807.

[6] Shimada, M. et al, 'Limiter Biasing Experiments in PBX Tokamak', Bull. Amer. Phys. Soc. 30 (1985) 1439.

[7] Conn. R.W, et al., ALT-I Pump Limiter Behavior and Edge Plasma Flows During Biasing and ICRF Heating in the TEXTOR Tokamak', Proceedings of 11th LAEA Conference on Plasma Physies and Controlled Nuclear Fusion Research, Kyoto, Vol. I (1987) p. 249.

[8] LaBombard, B., Lipschultz, B., 'Poloidal Asymmetries in the Scrape-off Layer Plasma of the Alcator C Tokamak', Nucl. Fusion 27 (1987) 81.

[9] Keilhacker, M., ct al., Nucl. Fusion 21 (1981) 1497.

[10] Itoh, S.I., Itoh, K., 'Model of $L$ - to $H$-Mode Transition in Tokamak', Phys. Rev. Lett. 30 (1988) 2276.

[11] Lipschultz, B., L.Bombard, B., Marmar, E.S., Pickrell, M.M., Terry, J.L., Watterson, R., Wolfe, S.M., TMARFE: An Edge Plasma Phenomenon', Nucl. Fusion 24 (1984) 977.

[12] Lipschultz, B., 'Review of Marfe Phenomena in Tokamaks', J. Nucl. Mater. 145-147 (1987) $15-25$.

[13] Vershkov, V.A., Grashin, S.A., Chankin, A.V., 'Experimental Study of Plasma Fluxes in the Shadow of a Scoop Limiter on T-10', J. Nucl. Mater. $145-147$ (1987) 611.

[14] Deboos, J.C., 'Confinement Studies in DWl-D', Bull. Amer. Phys. Soc. 32 (1987) 1711.

[15] Perkins, D.E., Ferguson, W., Shimada, M., Ali Mahdavi, M., Petrie, T.W., 'Divertor Langmuir Probe Measurements in DIII-D', Bull. Amer. Phys. Soc. 32 (1987) 1899.

[16] Tynan, G.R., LaBombard, B., Conn, R.W., Pribyl, P., Taylor, R.J., 'Poloidally Asymmetric Fluctuation Driven Particle Fluxes in the Edge Plasma of Ohmic Discharges', Bull. Amer. Phys. Soc. 33 (1988) 2051, and work currently in progress.

[17] Green, B.J., The Effect of Plasma Flow on Toroidal Confinement', Nucl. Fus. 12 (1972) 475. 


\section{Figure Captions}

Fig. 1 - Model Geometry for Parallel and Perpendicular Transport in a Biased Scrape-off Layer (BSOL). Limiter/divertor plates are biased to a potential, $\Phi_{W}$, which may be different than the floating potential, $\Phi_{1}$.

Fig. 2 - Scrape-off layer plasma density profile (from Lithium beam diagnostic) at $1.4 \mathrm{sec}$ into a TEXTOR discharge with positive bias, negative bias, or no bias applied to ALT-I [7]. The radial position of ALT-I and the main limiters are shown.

Fig. 3 - Two-zone biased scrape-off layer model for the results shown in fig. 2. Edge plasma connected along magnetic field lines to a negatively biased surface has a longer density e-folding length. Conversely, positively biased surfaces cause the local e-folding length io shorten.

Fig. 4 - $m=0$ and $m=1$ scrape-off layer biasing schemes. All 'limiter bias' experiments performed in tokamaks to date can be broadly classified in the $m=0$ category. In contrast, we propose the $m=1$ electrostatic barrier scrapeoff layer ( $m=1$ EBSOL) for limiter or divertor-based tokamaks. Conceptually, this biased scrape-off layer imposes an 'electrostatic barrier' to cross-field transport on the large major radius side of the tokamak.

Fig. 5 - ExB plasma flow patter for the $m=1$ EBSOL in a limiter and divertor system. Such flows may supplement or even eliminate the magnetic divertor system.

Fig. 6 - Two other 'electrostatic barrier' bias concepts used in conjunction with the $\mathrm{m}=1$ EBSOL. The divertor throats and wall surfaces may be biased positive with respect to the plasma, reducing the flux and energy of plasma ions striking these surfaces. Consequently, impurities originating from these surfaces may be greatly reduced while plasma exhaust control is independently maintained by the $m=1$ EBSOL scheme. 


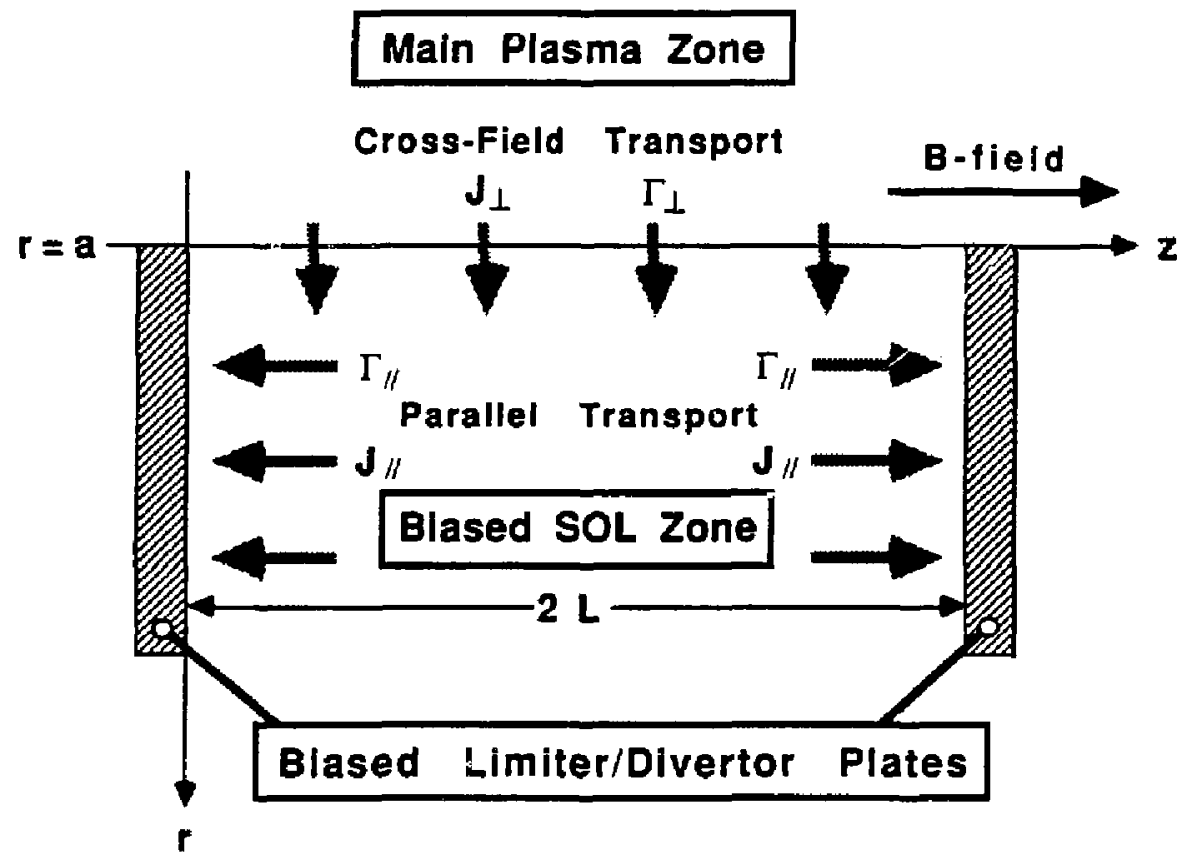

FIGURE 1 


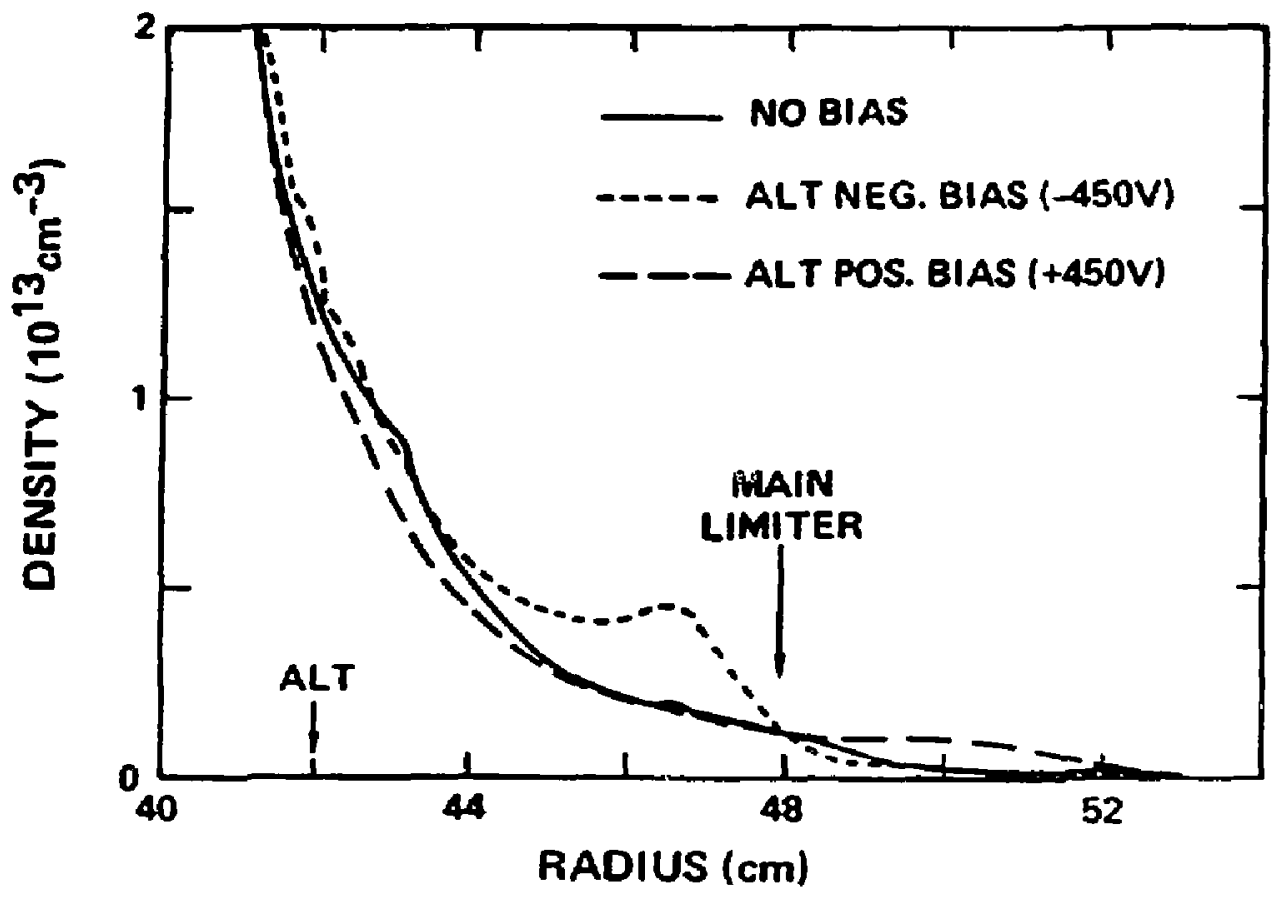

FIGURE 2 


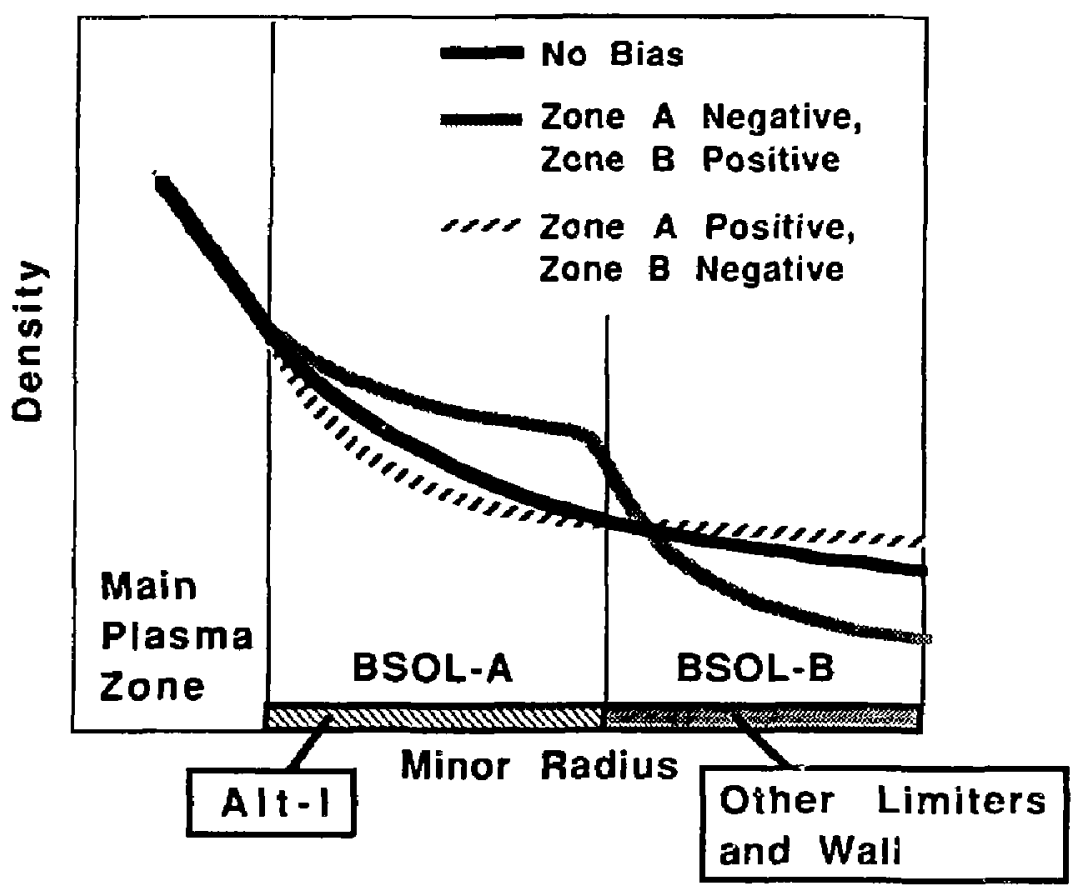

FIGURE 3 
a) $\mathrm{m}=0$ Biased Scrape-Off Laver

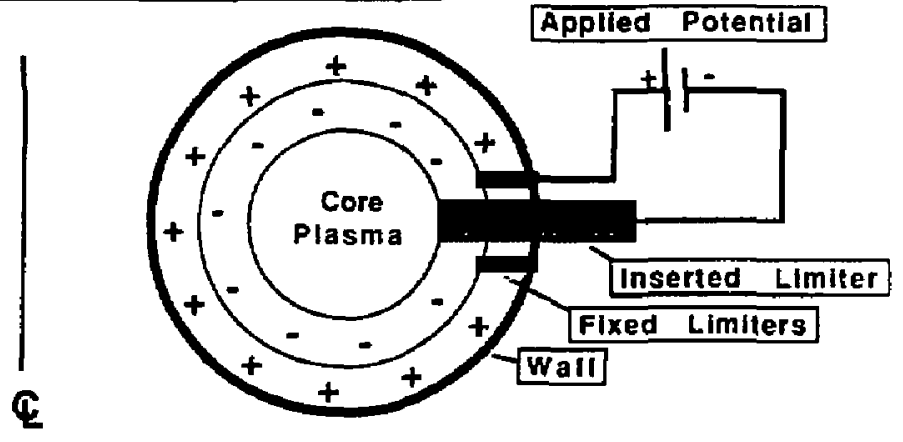

b) m=1 EBSOL - Limiter

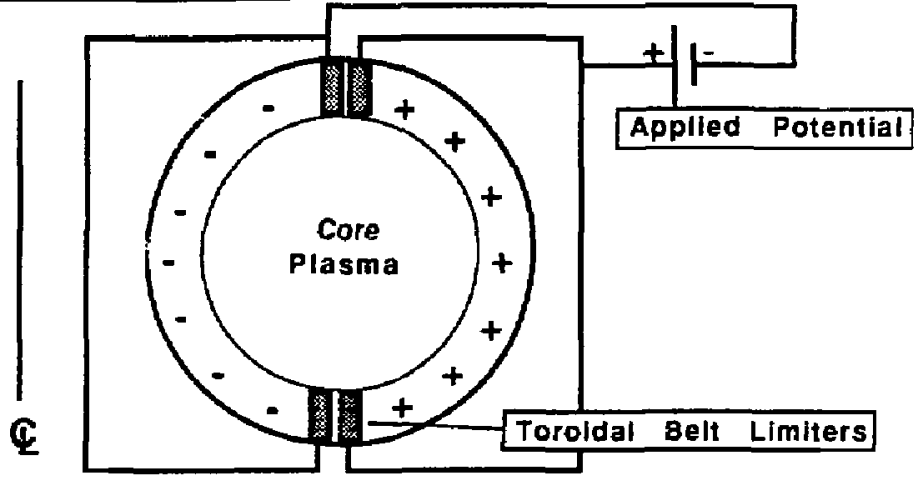

c) $m=1$ EBSOL - Divertor

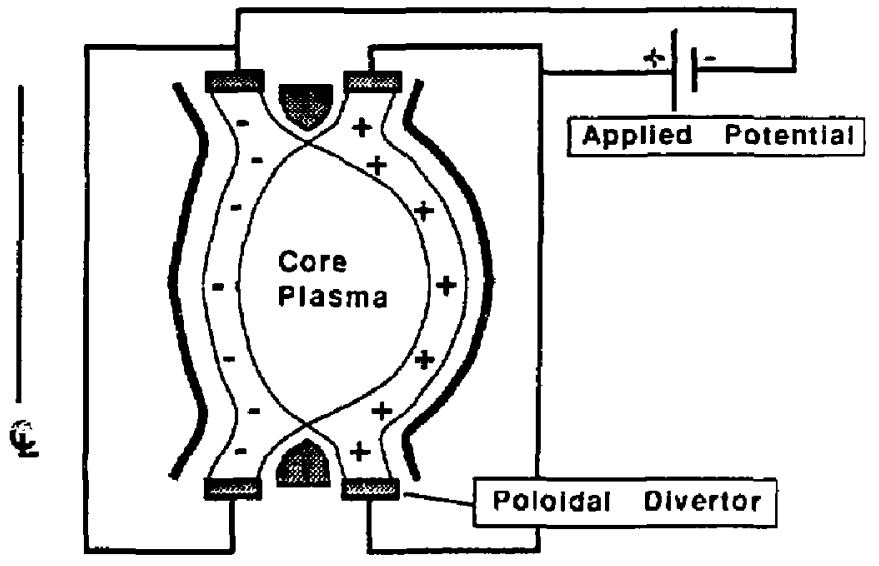

FIGURE 4 
a) Ey. Flows in $m=1$ EBSOL - Limiter

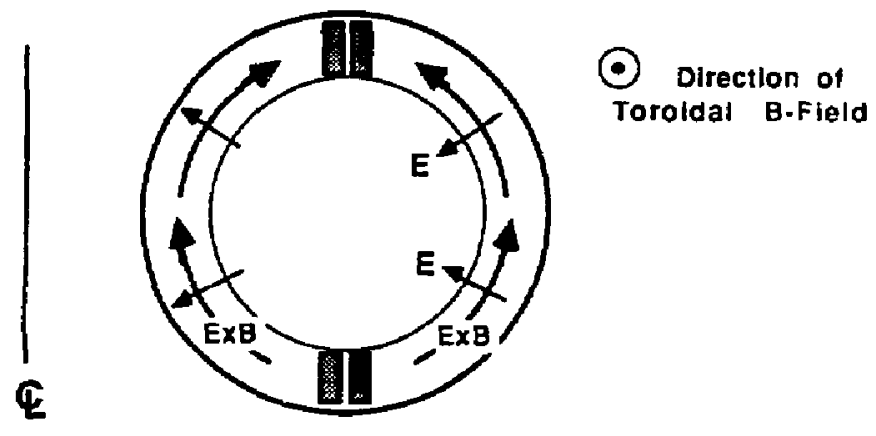

b) ExB Flows in $m=1$ EBSOL - Divertor

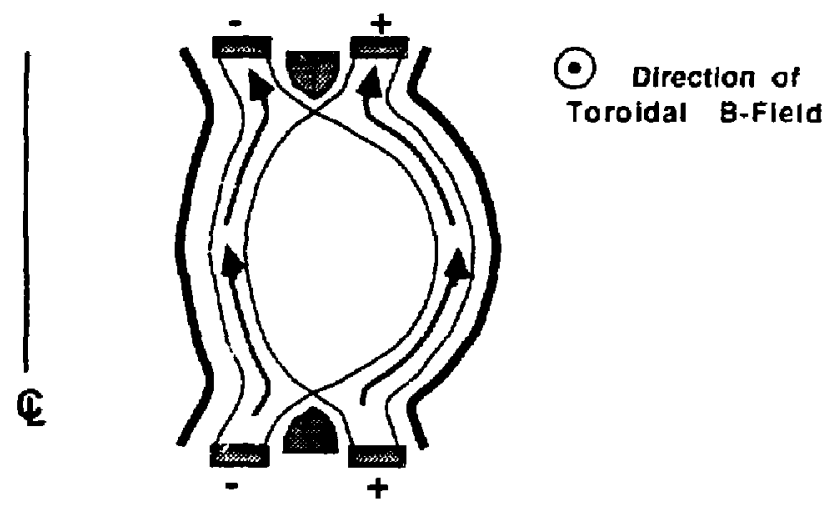

FIGURE 5 


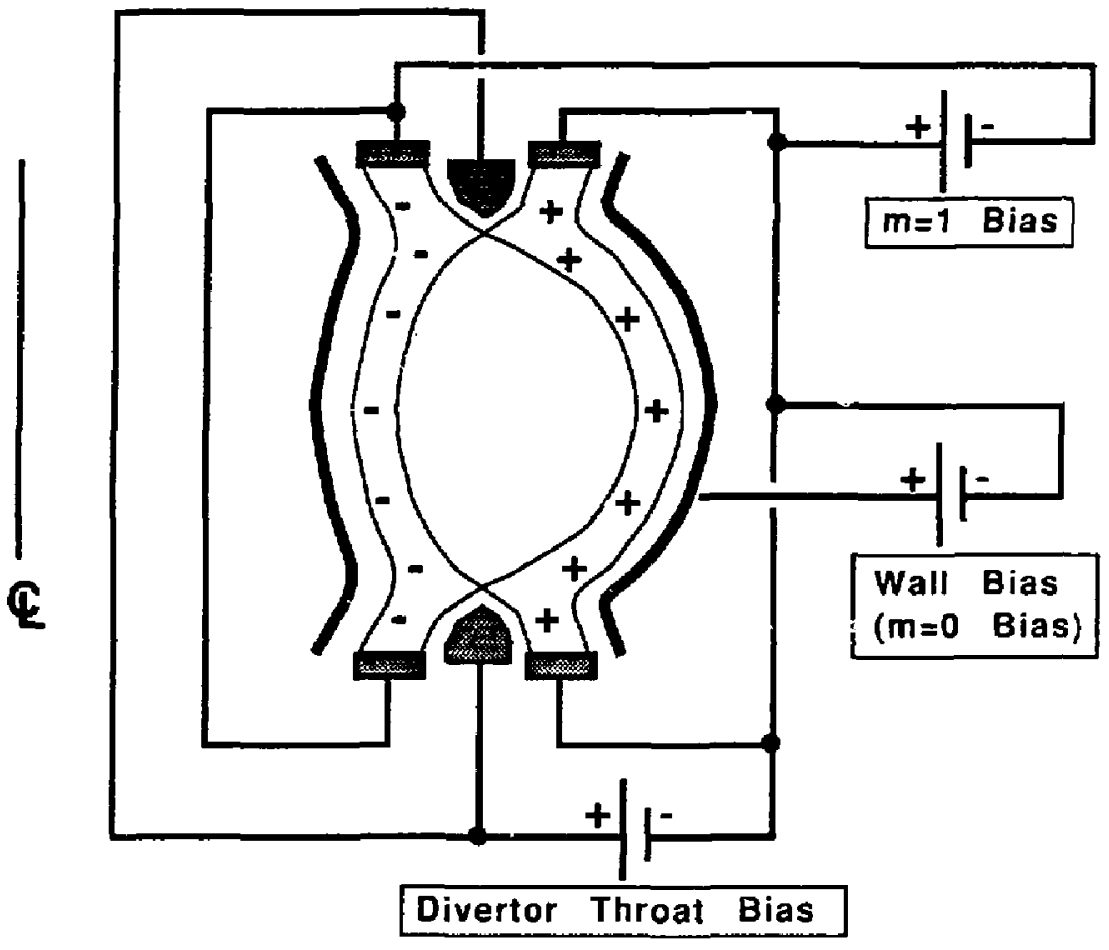

FIGURE 6 